\title{
A Class of Vector Lyapunov Functions for Stability Analysis of Nonlinear Impulsive Differential Systems
}

\author{
Zhang Qunli \\ Department of Mathematics, Heze University, Heze, Shandong 274015, China \\ Correspondence should be addressed to Zhang Qunli; qunli-zhang@126.com \\ Received 17 January 2014; Accepted 10 April 2014; Published 5 May 2014 \\ Academic Editor: Huaiqin Wu
}

Copyright ( 2014 Zhang Qunli. This is an open access article distributed under the Creative Commons Attribution License, which permits unrestricted use, distribution, and reproduction in any medium, provided the original work is properly cited.

A novel and effective approach to stability of the solutions of nonlinear systems with impulsive effect is considered. The investigations are carried out by means of a class of vector Lyapunov functions and differential inequalities for piecewise continuous functions. Simulation examples are given to illustrate the presented results.

\section{Introduction}

There has been a significant development in the theory of the impulsive differential systems within the past 20 years (see [1-17]). The efficient applications of impulsive differential systems require the finding of criteria for stability of their solutions.

It is well known that the more complex the considered dynamical system is, the more difficult it is to find a Lyapunov function. This leads to employing several Lyapunov functions, which is called to be a vector Lyapunov function, for which each component provides information about a part of the dynamics. Then some of the complex aspects can be reduced to the connected dynamical systems and less rigid requirements can be satisfied. Hence, the corresponding theory, namely, the method of vector Lyapunov functions, offers a very flexible process (see $[1-5,11,13,18]$ ).

In this paper, without assuming Lipschitz conditions on these nonlinear functions, which play important roles in the considered systems, as needed in most other papers, we will use a class of vector Lyapunov functions, by means of the comparison principle (see $[1,2,13]$ ), to study global exponential stability of the solution of nonlinear impulsive differential systems.

\section{Statement of the Problem}

Let $R^{n}$ be the $n$-dimensional Euclidean space with norm $\|x\|=$ $\sqrt{x^{T} x}=\sqrt{\langle x, x\rangle}=\left(\sum_{i=1}^{n} x_{i}^{2}\right)^{1 / 2}, x=\left(x_{1}, x_{2}, \ldots, x_{n}\right)^{T}$, let
$\Omega$ be a bounded domain in $R^{m}$ containing the origin, and $\langle u, v\rangle=u_{1} v_{1}+u_{2} v_{2}+\cdots+u_{n} v_{n}$, where $u=\left(u_{1}, u_{2}, \ldots, u_{n}\right)^{T}$, $v=\left(v_{1}, v_{2}, \ldots, v_{n}\right)^{T}$, and $R_{+}=[0,+\infty)$.

Let $t_{0} \in R_{+}$. Consider the impulsive functional differential system

$$
\begin{gathered}
\dot{x}(t)=f(t, x(t)), \quad\left(t \geq t_{0}, t \neq t_{k}, k=1,2,3, \ldots\right) \\
\Delta x=x\left(t_{k}+0\right)-x\left(t_{k}\right)=I_{k} x\left(t_{k}\right), \quad\left(t=t_{k}>t_{0}\right),
\end{gathered}
$$

where $f:\left(t_{0},+\infty\right) \times \Omega \rightarrow R^{n}, I_{k}: \Omega \rightarrow \Omega, k=1,2,3, \ldots$, $t_{0}<t_{1}<t_{2}<\cdots$, and $\lim _{k \rightarrow+\infty} t_{k}=+\infty$.

Definition 1 (see $[12,14,15,19]$ ). System (1) is called to be exponentially stable on a neighborhood $\Omega$ of the equilibrium point, if there exist constants $\mu>0, M>0$ such that

$$
\|x(t)\| \leq M e^{-\mu t}\left\|x_{0}\right\|, \quad(t \geq 0),
$$

where $x(t)$ is any solution of (1) initiated from $x\left(t_{0}\right)=x_{0}$.

We introduce a kind of partial ordering defined in the following natural way. For $u, v \in R^{n}, u \geq v(u>v)$ if and only if $u_{j} \geq v_{j}\left(u_{j}>v_{j}\right)$ for any $j=1,2, \ldots, n$.

Definition 2 (see $[1,2,13]$ ). The function $\Psi: \Omega \rightarrow R^{n}$ is called monotonically increasing function in $\Omega$ if $\Psi(u)>\Psi(v)$ for $u>v$ and $\Psi(u) \geq \Psi(v)$ for $u \geq v, u, v \in \Omega$.

Definition 3 (see $[1,2,13]$ ). The function $F: R_{+} \times \Omega \rightarrow R^{n}$ is called quasi-monotonically increasing function in $R_{+} \times \Omega$ 
if, for any two points $(t, u)$ and $(t, v)$ of $R_{+} \times \Omega$ and for any $i \in 1,2, \ldots, n$, the inequality $F_{i}(t, u) \geq F_{i}(t, v)$ holds always when $u_{i}=v_{i}$ and $u \geq v$, that is, if, for any $t \in R_{+}$fixed and any $i \in 1,2, \ldots, n$, the function $F_{i}(t, u)$ is nondecreasing with respect to $\left(u_{1}, \ldots, u_{i-1}, u_{i+1}, \ldots, u_{n}\right)$.

In the further considerations we will use the class $v_{0}$ of piecewise continuous auxiliary functions $V:\left[t_{0},+\infty\right) \times \Omega \rightarrow$ $R_{+}^{m}$ which are analogues of Lyapunov functions (see [20]).

We denote by $\kappa$ the class of all continuous and strictly increasing functions $a: R_{+} \rightarrow R_{+}$such that $a(0)=0$.

We are interested in focusing on the possibility of applying the comparison method to the stability of the zero solution of system (1). Together with the system (1), we will also consider the following comparison system:

$$
\begin{gathered}
\dot{u}(t)=g(t, u(t)), \quad\left(t \geq t_{0}, t \neq t_{k}, k=1,2,3, \ldots\right) \\
\Delta u=u\left(t_{k}+0\right)-u\left(t_{k}\right)=B_{k} u\left(t_{k}\right), \quad\left(t=t_{k}>t_{0}\right),
\end{gathered}
$$

where $g: R_{+} \times S(\rho) \rightarrow R^{m}, B_{k}: S(\rho) \rightarrow R^{m}, k=1,2,3, \ldots$, $t_{0}<t_{1}<t_{2}<\cdots, \lim _{k \rightarrow+\infty} t_{k}=+\infty$, and $S(\rho)=\left\{x \in R^{n}\right.$ : $\|x\|<\rho\}$ is an open subset of $R^{m}$.

Lemma 4 (see $[1,2,13]$ ). Let the following conditions be fulfilled:

(1) $V: R_{+} \times S(\rho) \rightarrow R_{+}^{m}, V \in \nu_{0}$,

$D^{+} V(t, x) \leq g(t, V(t, x)), \quad t \neq t_{k}, \quad(t, x) \in R_{+} \times S(\rho)$,

where $g: R_{+} \times R_{+}^{m} \rightarrow R_{+}^{m}$ is continuous, quasimonotonically increasing in $\left(t_{k-1}, t_{k}\right] \times R^{m}$ and $g(t, 0)=$ 0 for $t \in R_{+}$and $D^{+} V(t, x)=\lim _{h \rightarrow 0^{+}} \sup (1 / h)[V(t+$ $h, x+h f(t, x))-V(t, x)]$;

(2) there exists a $\rho_{0}$ which satisfies when $x_{0} \in S\left(\rho_{0}\right), u+$ $B_{k}(u)$ are monotonically increasing in $S(\rho)$ and $B_{k}(0)=$ 0 ;

(3) there exist the functions $a(\cdot), b(\cdot) \in \kappa$ which satisfy $b(\|x\|) \leq L(t, x) \leq a(\|x\|)$ for all $(t, x) \in$ $R_{+} \times S(\rho)$, where $L(t, x)=\sum_{i=1}^{m} V_{i}(t, x), V(t, x)=$ $\left(V_{1}(t, x), V_{2}(t, x), \ldots, V_{m}(t, x)\right)^{T}$.

If the zero solution of system (3) is stable, then the zero solution of system (1) is stable with respect to $x(t)$.

\section{Theory Results of Impulsive Stability}

3.1. Basic Results. Consider the following system

$$
\begin{aligned}
& \dot{x}(t)=F(t, x(t), y(t)), \\
& \dot{y}(t)=G(t, x(t), y(t)),
\end{aligned}
$$

where $x(t) \in R^{m}, y(t) \in R^{m}$, and $F(t, 0,0)=G(t, 0,0)=0$.

We will say that condition $(A)$ is satisfied if the following conditions hold.
(A) The functions $F(t, x(t), y(t)), G(t, x(t), y(t))$ are continuous in $\left(t_{k-1}, t_{k}\right] \times R^{m} \times R^{m}$. There exists a function $h(t)$, and the condition

$$
\frac{(\alpha F(t, x(t), y(t))+\beta G(t, x(t), y(t)))^{T}(\alpha x(t)+\beta y(t))}{\|\alpha x(t)+\beta y(t)\|^{2}}
$$$$
\leq h(t)
$$

holds for any $\alpha, \beta$.

We will say that condition (B) is satisfied if the following conditions hold.

(B) The functions $F_{i}(t, x(t), y(t)), G_{i}(t, x(t), y(t))$ are continuous in $\left(t_{k-1}, t_{k}\right] \times R^{m} \times R^{m}$. There exist functions $h_{j i}(t)$, and the condition

$$
\begin{aligned}
& \left(\left(\alpha_{j} F_{i}(t, x(t), y(t))+\beta_{j} G_{i}(t, x(t), y(t))\right)^{T}\right. \\
& \left.\quad \times\left(\alpha_{j} x(t)+\beta_{j} y(t)\right)\right) \\
& \quad \times\left(\left\|\alpha_{j} x(t)+\beta_{j} y(t)\right\|\left\|\alpha_{i} x(t)+\beta_{i} y(t)\right\|\right)^{-1} \\
& \leq h_{j i}(t)
\end{aligned}
$$

holds for any $\alpha_{j}, \beta_{j}, \alpha_{i}, \beta_{i}$.

We will say that condition $(\mathrm{C})$ is satisfied if the following conditions hold.

(C) The functions $F_{i}(t, x(t), y(t)), G_{i}(t, x(t), y(t))$ are continuous in $\left(t_{k-1}, t_{k}\right] \times R^{m} \times R^{m}$. There exist functions $h_{j i}(t)$, and the condition

$$
\begin{aligned}
& \left(\left(\alpha_{j} F_{i}(t, x(t), y(t))+\beta_{j} G_{i}(t, x(t), y(t))\right)^{T}\right. \\
& \left.\quad \times\left(\alpha_{j} x(t)+\beta_{j} y(t)\right)\right) \\
& \quad \times\left(\left\|\alpha_{j} x(t)+\beta_{j} y(t)\right\|\left\|\alpha_{i} x(t-\tau)+\beta_{i} y(t-\tau)\right\|\right)^{-1} \\
& \quad \leq s_{j i}(t)
\end{aligned}
$$

holds for any $\alpha_{j}, \beta_{j}, \alpha_{i}, \beta_{i}$.

Theorem 5. If the operators $F, G$ in system (5) satisfy condition (A) for any $x, y \in \Omega$, then $x(t)$ and $y(t)$ initiated from $x\left(t_{0}\right)=$ $x_{0} \in \Omega, y\left(t_{0}\right)=y_{0} \in \Omega$ satisfy

$$
(\|\alpha x(t)+\beta y(t)\|)_{t}^{\prime} \leq h(t)\|\alpha x(t)+\beta y(t)\| \quad \forall t \geq 0 .
$$

Proof. For all $t \geq 0$ and $r \geq 0$, we have

$$
\begin{aligned}
\frac{d \alpha e^{r t} x(t)}{d t} & =\alpha r e^{r t} x(t)+\alpha e^{r t} \dot{x}(t) \\
& =\alpha r e^{r t} x(t)+\alpha e^{r t} F(t, x(t), y(t)), \\
\frac{d \beta e^{r t} y(t)}{d t} & =\beta r e^{r t} y(t)+\beta e^{r t} \dot{y}(t) \\
& =\beta r e^{r t} y(t)+\beta e^{r t} G(t, x(t), y(t)),
\end{aligned}
$$




$$
\begin{aligned}
& \frac{d \alpha e^{r t} x(t)}{d t}+\frac{d \beta e^{r t} y(t)}{d t} \\
& =\frac{d\left(\alpha e^{r t} x(t)+\beta e^{r t} y(t)\right)}{d t}=\frac{d\left[e^{r t}(\alpha x(t)+\beta y(t))\right]}{d t} \\
& =e^{r t}[\alpha F(t, x(t), y(t))+\beta G(t, x(t), y(t)) \\
& \quad+\alpha r x(t)+\beta r y(t)], \\
& e^{r t}(\alpha x(t)+\beta y(t)) \\
& =e^{r s}(\alpha x(s)+\beta y(s)) \\
& \quad+\int_{s}^{t} e^{r u}[\alpha F(u, x(u), y(u))+\beta G(u, x(u), y(u)) \\
& \quad+\alpha r x(u)+\beta r y(u)] d u, \\
& e^{r t}\|\alpha x(t)+\beta y(t)\|-e^{r s}\|\alpha x(s)+\beta y(s)\| \\
& \leq \int_{s}^{t} e^{r u} \| \alpha F(u, x(u), y(u))+\beta G(u, x(u), y(u)) \\
& \quad+\alpha r x(u)+\beta r y(u) \| d u .
\end{aligned}
$$

The derivative with respect to time $t$ is

$$
\begin{gathered}
e^{r t}(\|\alpha x(t)+\beta y(t)\|)_{t}^{\prime}+r e^{r t}\|\alpha x(t)+\beta y(t)\| \\
\leq e^{r t} \| \alpha F(t, x(t), y(t))+\beta G(t, x(t), y(t)) \\
+\alpha r x(t)+\beta r y(t) \| .
\end{gathered}
$$

That is,

$$
\begin{aligned}
& (\|\alpha x(t)+\beta y(t)\|)_{t}^{\prime} \\
& \leq \| \alpha F(t, x(t), y(t))+\beta G(t, x(t), y(t)) \\
& \quad+\alpha r x(t)+\beta r y(t)\|-r\| \alpha x(t)+\beta y(t) \| .
\end{aligned}
$$

It is easy to prove that $\| \alpha F(t, x(t), y(t))+\beta G(t, x(t), y(t))+$ $\alpha r x(t)+\beta r y(t)\|-r\| \alpha x(t)+\beta y(t) \|$ is monotonically decreasing on $r$; thus the limit

$$
\begin{aligned}
\lim _{r \rightarrow+\infty}( & \| \alpha F(t, x(t), y(t))+\beta G(t, x(t), y(t)) \\
& +\alpha r x(t)+\beta r y(t)\|-r\| \alpha x(t)+\beta y(t) \|)
\end{aligned}
$$

exists. From the norm $\|x\|=\sqrt{x^{T} x}=\sqrt{\langle x, x\rangle}=\left(\sum_{i=1}^{n} x_{i}^{2}\right)^{1 / 2}$ and condition (A), we obtain

$$
\begin{aligned}
& (\|\alpha x(t)+\beta y(t)\|)_{t}^{\prime} \\
& \leq[(\alpha F(t, x(t), y(t))+\beta G(t, x(t), y(t))+\alpha r x(t) \\
& +\beta r y(t))^{T}(\alpha F(t, x(t), y(t))+\beta G(t, x(t), y(t)) \\
& +\alpha r x(t)+\beta r y(t))]^{1 / 2} \\
& -r\left((\alpha x(t)+\beta y(t))^{T}(\alpha x(t)+\beta y(t))\right)^{1 / 2}
\end{aligned}
$$

$$
\begin{aligned}
= & \frac{q(t, \alpha, \beta)}{(p(t, \alpha, \beta))^{1 / 2}+r\|\alpha x(t)+\beta y(t)\|} \\
= & \frac{q(t, \alpha, \beta) / r}{(p(t, \alpha, \beta))^{1 / 2} / r+\|\alpha x(t)+\beta y(t)\|} \\
\leq & \lim _{r \rightarrow+\infty} \frac{q(t, \alpha, \beta) / r}{(p(t, \alpha, \beta))^{1 / 2} / r+\|\alpha x(t)+\beta y(t)\|} \\
= & \frac{(\alpha F(t, x(t), y(t))+\beta G(t, x(t), y(t)))^{T}(\alpha x(t)+\beta y(t))}{\|\alpha x(t)+\beta y(t)\|} \\
= & \frac{(\alpha F(t, x(t), y(t))+\beta G(t, x(t), y(t)))^{T}(\alpha x(t)+\beta y(t))}{\|\alpha x(t)+\beta y(t)\|^{2}} \\
& \times\|\alpha x(t)+\beta y(t)\| \\
\leq & h(t)\|\alpha x(t)+\beta y(t)\|,
\end{aligned}
$$

where $q(t, \alpha, \beta)=\|\alpha F(t, x(t), y(t))+\beta G(t, x(t), y(t))\|^{2}$ $+2 r(\alpha F(t, x(t), y(t))+\beta G(t, x(t), y(t)))^{T}(\alpha x(t)+\beta y(t))$ and $p(t, \alpha, \beta)=\|\alpha F(t, x(t), y(t))+\beta G(t, x(t), y(t))\|^{2}+$ $2 r(\alpha F(t, x(t), y(t))+\beta G(t, x(t), y(t)))^{T}(\alpha x(t)+\beta y(t))+$ $r^{2}\|\alpha x(t)+\beta y(t)\|^{2}$.

Namely,

$$
(\|\alpha x(t)+\beta y(t)\|)_{t}^{\prime} \leq h(t)\|\alpha x(t)+\beta y(t)\| .
$$

Corollary 6. When $x(t) \in R^{n}, y(t) \in R^{m}$, and $n>m$, let

$$
y(t) \longrightarrow\left(\begin{array}{c}
y(t) \\
0_{n-m}
\end{array}\right)=\tilde{y}(t),
$$

$$
G(t, x(t), y(t))
$$

$$
\longrightarrow\left(\begin{array}{c}
G(t, x(t), y(t)) \\
0_{n-m}
\end{array}\right)=\widetilde{G}(t, x(t), \tilde{y}(t)),
$$

and $\quad(\alpha F(t, x(t), \widetilde{y}(t))+\beta \widetilde{G}(t, x(t), \widetilde{y}(t)))^{T}(\alpha x(t) \quad+$ $\beta \tilde{y}(t)) /\|\alpha x(t)+\beta \widetilde{y}(t)\|^{2} \leq h(t)$ holds for any $\alpha, \beta$, where $0_{n-m}=(0,0, \ldots, 0)^{T} \in R^{n-m}$. Then one obtains a similar result with Theorem 5:

$$
(\|\alpha x(t)+\beta \tilde{y}(t)\|)_{t}^{\prime} \leq h(t)\|\alpha x(t)+\beta \tilde{y}(t)\| \quad \forall t \geq 0 .
$$

Corollary 7. When $x(t) \in R^{n}, y(t) \in R^{m}$, and $n>m$, let

$$
\begin{aligned}
x(t) \longrightarrow\left(\begin{array}{c}
x(t) \\
0_{1}
\end{array}\right)=\tilde{x}(t), \quad y(t) \longrightarrow\left(\begin{array}{c}
y(t) \\
0_{2}
\end{array}\right)=\tilde{y}(t), \\
F(t, x(t), y(t)) \\
\longrightarrow\left(\begin{array}{c}
F(t, x(t), y(t)) \\
0_{1}
\end{array}\right)=\widetilde{F}(t, \tilde{x}(t), \tilde{y}(t)), \\
G(t, x(t), y(t)) \\
\longrightarrow\left(\begin{array}{c}
G(t, x(t), y(t)) \\
0_{2}
\end{array}\right)=\widetilde{G}(t, \tilde{x}(t), \tilde{y}(t)),
\end{aligned}
$$


and $(\alpha \widetilde{F}(t, \widetilde{x}(t), \widetilde{y}(t))+\beta \widetilde{G}(t, \widetilde{x}(t), \widetilde{y}(t)))^{T}(\alpha \widetilde{x}(t)+\beta \widetilde{y}(t)) /$ $\|\alpha \tilde{x}(t)+\beta \tilde{y}(t)\|^{2} \leq h(t)$ holds for any $\alpha, \beta$, where $0_{1}=$ $(0,0, \ldots, 0)^{T} \in R^{m}, 0_{2}=(0,0, \ldots, 0)^{T} \in R^{n}$. Then one obtains a similar result with Theorem 5 :

$$
(\|\alpha \tilde{x}(t)+\beta \tilde{y}(t)\|)_{t}^{\prime} \leq h(t)\|\alpha \widetilde{x}(t)+\beta \tilde{y}(t)\| \quad \forall t \geq 0 .
$$

Remark 8. When $\alpha=1, \beta=-1$, and $F=G$, the constant

$$
\sup _{x, y \in \Omega, x \neq y} \frac{(F(x(t))-F(y(t)))^{T}(x(t)-y(t))}{\|x(t)-y(t)\|^{2}}
$$

is called the nonlinear measure [21-26]. But the condition

$$
\begin{aligned}
& \frac{(\alpha F(t, x(t), y(t))+\beta G(t, x(t), y(t)))^{T}(\alpha x(t)+\beta y(t))}{\|\alpha x(t)+\beta y(t)\|^{2}} \\
& \quad \leq h(t)
\end{aligned}
$$

is weaker than the nonlinear measure.

When system (5) is written as

$$
\begin{aligned}
& \dot{x}(t)=F_{1}(t, x(t), y(t))+F_{2}(t, x(t), y(t)), \\
& \dot{y}(t)=G_{1}(t, x(t), y(t))+G_{2}(t, x(t), y(t)),
\end{aligned}
$$

where $x(t) \in R^{m}, y(t) \in R^{m}$, and $F_{i}(t, 0,0)=G_{i}(t, 0,0)=0$, $i=1,2$, we have the following conclusion.

Corollary 9. If the operators $F_{1}, F_{2}, G_{1}, G_{2}$ in system (22) satisfy condition (B) for any $x, y \in \Omega$, then $x(t)$ and $y(t)$ initiated from $x\left(t_{0}\right)=x_{0} \in \Omega, y\left(t_{0}\right)=y_{0} \in \Omega$ satisfy

$$
\begin{array}{r}
\left(\left\|\alpha_{1} x(t)+\beta_{1} y(t)\right\|\right)_{t}^{\prime} \\
\leq h_{11}(t)\left\|\alpha_{1} x(t)+\beta_{1} y(t)\right\|+h_{12}(t)\left\|\alpha_{2} x(t)+\beta_{2} y(t)\right\| \\
\forall t \geq 0 .
\end{array}
$$

When system (5) is written as

$$
\begin{aligned}
\dot{x}(t) & =\sum_{i=1}^{s} F_{i}(t, x(t), y(t)), \\
\dot{y}(t) & =\sum_{i=1}^{s} G_{1}(t, x(t), y(t)),
\end{aligned}
$$

where $x(t) \in R^{m}, y(t) \in R^{m}$, and $F_{i}(t, 0,0)=G_{i}(t, 0,0)=0$, $i=1,2, \ldots, s$, we have the following conclusion.

Corollary 10. If the operators $F_{i}, G_{i}, i=1,2, \ldots, s$, in system (24) satisfy condition (B) for any $x, y \in \Omega$, then $x(t)$ and $y(t)$ initiated from $x\left(t_{0}\right)=x_{0} \in \Omega, y\left(t_{0}\right)=y_{0} \in \Omega$ satisfy

$$
\left(\left\|\alpha_{j} x(t)+\beta_{j} y(t)\right\|\right)_{t}^{\prime} \leq \sum_{i=1}^{s} h_{j i}(t)\left\|\alpha_{i} x(t)+\beta_{i} y(t)\right\| \quad \forall t \geq 0 .
$$

Corollary 11. If the operators $F_{i}, G_{i}, i=1,2, \ldots, s$, in system (24) satisfy condition (B) for any $x, y \in \Omega$, then $x(t)$ and $y(t)$ initiated from $x\left(t_{0}\right)=x_{0} \in \Omega, y\left(t_{0}\right)=y_{0} \in \Omega$ and the constant $\sigma$ satisfy

$$
\left(e^{\sigma t}\left\|\alpha_{j} x(t)+\beta_{j} y(t)\right\|\right)_{t}^{\prime} \leq \sum_{i=1}^{s} h_{j i}(t)\left(e^{\sigma t}\left\|\alpha_{i} x(t)+\beta_{i} y(t)\right\|\right)
$$

When system (5) is

$$
\begin{gathered}
\dot{x}(t)=F_{1}(t, x(t), y(t))+F_{2}(t, x(t-\tau), y(t-\tau)), \\
\dot{y}(t)=G_{1}(t, x(t), y(t))+G_{2}(t, x(t-\tau), y(t-\tau)),
\end{gathered}
$$

where $x(t) \in R^{m}, y(t) \in R^{m}, F_{i}(t, 0,0)=G_{i}(t, 0,0)=0$, $i=1,2$, and $\tau>0$ is the time delay, we have the following conclusion.

Corollary 12. If the operators $F_{1}, F_{2}, G_{1}, G_{2}$ in system (27) satisfy conditions $(B)$ and $(C)$ for any $x, y \in \Omega$, then $x(t)$ and $y(t)$ initiated from $x\left(t_{0}\right)=x_{0} \in \Omega, y\left(t_{0}\right)=y_{0} \in \Omega$ satisfy

$$
\begin{aligned}
&\left(\left\|\alpha_{1} x(t)+\beta_{1} y(t)\right\|\right)_{t}^{\prime} \\
& \leq h_{11}(t)\left\|\alpha_{1} x(t)+\beta_{1} y(t)\right\| \\
&+s_{11}(t)\left\|\alpha_{1} x(t-\tau)+\beta_{1} y(t-\tau)\right\| \\
& \forall t \geq 0 .
\end{aligned}
$$

When system (5) is written as

$$
\begin{aligned}
& \dot{x}(t)=F(t, x(t), y(t)), \\
& \dot{y}(t)=G(t, x(t), y(t)), \\
& \dot{z}(t)=H(t, x(t), y(t)),
\end{aligned}
$$

where $x(t) \in R^{m}, y(t) \in R^{m}, z(t) \in R^{m}$, and $F(t, 0,0)=$ $G(t, 0,0)=H(t, 0,0)=0$, we have the following conclusion.

Corollary 13. If the operators $F, G, H$ in system (29) satisfy the condition

$$
\begin{aligned}
& ((\alpha F(t, x(t), y(t))+\beta G(t, x(t), y(t)) \\
& \left.\quad+\eta H(t, x(t), y(t)))^{T}(\alpha x(t)+\beta y(t)+\eta z(t))\right) \\
& \quad \times\left(\|\alpha x(t)+\beta y(t)+\eta z(t)\|^{2}\right)^{-1} \\
& \quad \leq l(t)
\end{aligned}
$$

for any $\alpha, \beta, \eta$ and $x, y, z \in \Omega$, then $x(t), y(t)$, and $z(t)$ initiated from $x\left(t_{0}\right)=x_{0} \in \Omega, y\left(t_{0}\right)=y_{0} \in \Omega$, and $z\left(t_{0}\right)=z_{0} \in \Omega$ satisfy

$$
(\|\alpha x(t)+\beta y(t)+\eta z(t)\|)_{t}^{\prime} \leq l(t)\|\alpha x(t)+\beta y(t)+\eta z(t)\|
$$


3.2. Impulsive Stability. Consider the impulsive differential system

$$
\begin{gathered}
\dot{x}(t)=F(t, x(t), y(t)), \quad\left(t \geq t_{0}, t \neq t_{k}, k=1,2,3, \ldots\right) \\
\dot{y}(t)=G(t, x(t), y(t)), \quad\left(t \geq t_{0}, t \neq t_{k}, k=1,2,3, \ldots\right) \\
\Delta\left(x\left(t_{k}\right), y\left(t_{k}\right)\right)^{T} \\
=\left(x\left(t_{k}+0\right)-x\left(t_{k}\right), y\left(t_{k}+0\right)-y\left(t_{k}\right)\right)^{T} \\
=I_{k}\left(x\left(t_{k}\right), y\left(t_{k}\right)\right), \quad\left(t=t_{k}>t_{0}\right),
\end{gathered}
$$

where $F, G:\left(t_{0},+\infty\right) \times \Omega \times \Omega \rightarrow R^{m}, I_{k}: \Omega \times \Omega \rightarrow \Omega \times \Omega$, $k=1,2,3, \ldots, t_{0}<t_{1}<t_{2}<\cdots$, and $\lim _{k \rightarrow+\infty} t_{k}=+\infty$.

Theorem 14. If the operators F, $G$ in system (32) and the constants $\alpha_{i}, \beta_{i}, i=1,2$, satisfy condition $(B)$ for any $x, y \in \Omega$ and the matrix $\left(\begin{array}{ll}\alpha_{1} & \beta_{1} \\ \alpha_{2} & \beta_{2}\end{array}\right)$ is invertible,

$$
\left(\begin{array}{lll}
h_{11}(t) & h_{12}(t) \\
h_{21}(t) & h_{22}(t)
\end{array}\right)\left(\begin{array}{l}
\left\|\alpha_{1} x(t)+\beta_{1} y(t)\right\| \\
\left\|\alpha_{2} x(t)+\beta_{2} y(t)\right\|
\end{array}\right)
$$

is continuous, quasi-monotonically increasing in $\left(t_{k-1}, t_{k}\right] \times R^{m}$,

$$
\left\|\left(\begin{array}{ll}
\alpha_{i} & \beta_{i}
\end{array}\right)\left(\left(\begin{array}{l}
x(t) \\
y(t)
\end{array}\right)+I_{k}\left(x\left(t_{k}\right), y\left(t_{k}\right)\right)\right)\right\|
$$

are monotonically increasing, $i=1,2$, and $I_{k}(0,0)=(0,0)^{T}$. If the zero solution of system (3) is stable, $u(t)=\left(\begin{array}{l}u_{1}(t) \\ u_{2}(t)\end{array}\right), u_{i}(t)=$ $\left\|\alpha_{i} x(t)+\beta_{i} y(t)\right\|$, and $i=1,2, B_{k} u(t)=\left(\| \alpha_{1} x(t)+\beta_{1} y(t)+\right.$ $\left.I_{k}\left(x\left(t_{k}\right), y\left(t_{k}\right)\right)\|,\| \alpha_{2} x(t)+\beta_{2} y(t)+I_{k}\left(x\left(t_{k}\right), y\left(t_{k}\right)\right) \|\right)^{T}-u(t)$, then the zero solution of system (32) is also stable.

Proof. From Corollary 10, we can obtain

$$
\begin{aligned}
&\left(\left\|\alpha_{1} x(t)+\beta_{1} y(t)\right\|\right)_{t}^{\prime} \\
& \leq h_{11}(t)\left\|\alpha_{1} x(t)+\beta_{1} y(t)\right\|+h_{12}(t)\left\|\alpha_{2} x(t)+\beta_{2} y(t)\right\| \forall t \geq 0, \\
&\left(\left\|\alpha_{2} x(t)+\beta_{2} y(t)\right\|\right)_{t}^{\prime} \\
& \quad \leq h_{21}(t)\left\|\alpha_{1} x(t)+\beta_{1} y(t)\right\|+h_{22}(t)\left\|\alpha_{2} x(t)+\beta_{2} y(t)\right\|
\end{aligned}
$$

It is easy to verify that all the conditions in the lemma are satisfied when $V=\left(u_{1}(t), u_{2}(t)\right)^{T}$. So it is true that the zero solution of system (32) is stable.

Remark 15. Imitating Theorem 14 and combining the above 7 corollaries, respectively, we can obtain similar results with Theorem 14 , respectively.

\section{Example}

Example 1. We consider the system $[3,13]$

$$
\begin{gathered}
\frac{d x}{d t}=e^{-t} x+y \sin t-\left(x^{3}+x y^{2}\right) \sin ^{2} t, \quad t \neq t_{k}, \\
\frac{d y}{d t}=x \sin t+e^{-t} y-\left(x^{2} y+y^{3}\right) \sin ^{2} t, \quad t \neq t_{k}, \\
\Delta x=a x+b y, \quad \Delta y=b x+a y, \quad t=t_{k},
\end{gathered}
$$

where $2 a=\sqrt{1+c_{1}}+\sqrt{1+c_{2}}-2,2 b=\sqrt{1+c_{1}}-\sqrt{1+c_{2}}$, $-1<c_{1} \leq 0$, and $-1<c_{2} \leq 0$. Consider

$$
\begin{aligned}
\alpha F( & t, x, y)+\beta G(t, x, y) \\
= & \alpha e^{-t} x+\alpha y \sin t-\alpha\left(x^{3}+x y^{2}\right) \sin ^{2} t+\beta x \sin t \\
& +\beta e^{-t} y-\beta\left(x^{2} y+y^{3}\right) \sin ^{2} t \\
= & e^{-t}(\alpha x+\beta y)+(\alpha y+\beta x) \sin t \\
& \quad-(\alpha x+\beta y)\left(x^{2}+y^{2}\right) \sin ^{2} t, \\
\langle\alpha F(t, x, y)+\beta G(t, x, y), \alpha x+\beta y\rangle & e^{-t}(\alpha x+\beta y)^{2}+(\alpha y+\beta x)(\alpha x+\beta y) \sin t \\
& \quad-(\alpha x+\beta y)^{2}\left(x^{2}+y^{2}\right) \sin ^{2} t, \\
\frac{(\alpha F(}{(t, x, y)+\beta G(t, x, y))^{T}(\alpha x+\beta y)} & \|\alpha x+\beta y\| \\
\leq & \frac{e^{-t}(\alpha x+\beta y)^{2}}{\|\alpha x+\beta y\|^{2}}\|\alpha x+\beta y\| \\
& +\frac{(\alpha y+\beta x)(\alpha x+\beta y) \sin t}{\|\alpha x+\beta y\|\|\alpha y+\beta x\|}\|\alpha y+\beta x\| \\
& e^{-t}\|\alpha x+\beta y\|+\|\alpha y+\beta x\||\sin t| .
\end{aligned}
$$

So $(\|\alpha x+\beta y\|)_{t}^{\prime} \leq e^{-t}\|\alpha x+\beta y\|+\|\alpha y+\beta x\||\sin t|, t \neq t_{k}$.

Similarly, $(\|\alpha y+\beta x\|)_{t}^{\prime} \leq e^{-t}\|\alpha y+\beta x\|+\| \alpha x+$ $\beta y \||\sin t|, t \neq t_{k}$.

When $\alpha x+\beta y=\varphi(\alpha y+\beta x)$ for all $x, y$, we can obtain $\varphi= \pm 1$. That is, $\alpha=\beta \neq 0$ and $\alpha=-\beta \neq 0$, respectively. Then we get the comparison system

$$
\begin{gathered}
\frac{d u_{1}}{d t}=e^{-t} u_{1}+|\sin t| u_{2}, \quad t \neq t_{k}, \\
\frac{d u_{2}}{d t}=|\sin t| u_{1}+e^{-t} u_{2}, \quad t \neq t_{k}, \\
u_{1}\left(t_{k}^{+}\right)=|(x+a x+b y)+(y+b x+a y)| \\
=\sqrt{1+c_{1}} u_{1}, \quad t=t_{k}, \\
u_{2}\left(t_{k}^{+}\right)=|(y+b x+a y)-(x+a x+b y)| \\
=\sqrt{1+c_{2}} u_{2}, \quad t=t_{k} .
\end{gathered}
$$


When $\alpha x+\beta y \neq \varphi(\alpha y+\beta x)$ for all $x, y$, we can obtain the comparison system

$$
\begin{gathered}
\frac{d u_{1}}{d t}=e^{-t} u_{1}+|\sin t| u_{2}, \quad t \neq t_{k}, \\
\frac{d u_{2}}{d t}=|\sin t| u_{1}+e^{-t} u_{2}, \quad t \neq t_{k}, \\
u_{1}\left(t_{k}^{+}\right)=|1+a| u_{1}+|b| u_{2}, \quad t=t_{k}, \\
u_{2}\left(t_{k}^{+}\right)=|b| u_{1}+|1+a| u_{2}, \quad t=t_{k} .
\end{gathered}
$$

We know that when the constants $\alpha, \beta$ satisfy the condition in Theorem 14, $u_{1}, u_{2}$ in the systems (38) and (39) are stable by stability theory of impulsive differential systems. So it is true that the zero solution of system (36) is stable. Taking $c_{1}=-0.2, c_{2}=-0.5$, and initial conditions $(x, y)^{T}=(1,2)^{T}$, the stability simulations of the states $x(t), y(t)$ of system (36) are as shown in Figures 1 and 2, respectively.

Example 2. We consider the system [3]

$$
\begin{gathered}
\frac{d x}{d t}=-2 x\left(y^{2}-(2+\sin t) x\right)^{2}-\frac{x \cos t}{2+\sin t}, \quad t \neq t_{k}, \\
\frac{d y}{d t}=-y\left(y^{2}-(2+\sin t) x\right)^{2}, \quad t \neq t_{k}, \\
\Delta x=\left(\frac{-1}{2}\right) x, \quad \Delta y=\left(\frac{1}{\sqrt{2}}-1\right), \quad t=t_{k} .
\end{gathered}
$$

Combining Corollary 11 and imitating Example 1, we can obtain the comparison system

$$
\begin{gathered}
\frac{d u_{1}}{d t}=\left(\sigma+\left|\frac{\cos t}{2+\sin t}\right|\right) u_{1}, \quad t \neq t_{k}, \\
\frac{d u_{2}}{d t}=\sigma u_{2}, \quad t \neq t_{k}, \\
u_{1}\left(t_{k}^{+}\right)=\left(\frac{1}{2}\right) u_{1}, \quad u_{2}\left(t_{k}^{+}\right)=\left(\frac{1}{\sqrt{2}}\right) u_{2}, \quad t=t_{k},
\end{gathered}
$$

where $u_{1}=e^{\sigma t}|\alpha x|, u_{2}=e^{\sigma t}|\beta y|$, and $0 \leq \sigma<1$. When the constants $\alpha, \beta$ satisfy the condition in Theorem 14 , it is true that the zero solution of system (40) is stable. Taking initial conditions $(x, y)^{T}=(1,2)^{T}$, the stability simulations of the states $x(t), y(t)$ of system (40) are as shown in Figures 3 and 4 , respectively.

Remark 16. Comparing Examples 1 and 2 in this paper with Examples 2 and 4 in the literature $[3,13]$ or [3], the vector Lyapunov functions $V(t, x, y)=\left(\begin{array}{c}|\alpha x+\beta y| \\ |\beta x+\alpha y|\end{array}\right), V(t, x, y)=$ $\left(\begin{array}{c}e^{\sigma t}|\alpha x| \\ e^{\sigma t}|\beta y|\end{array}\right)$ in this paper are simpler than the vector Lyapunov functions $V(t, x, y)=\left(\begin{array}{c}(x+y)^{2} \\ (x-y)^{2}\end{array}\right), V(t, x, y)=\left(y^{2}-(2+\sin t) x\right)^{2}$ in $[3,13]$ or $[3]$, respectively, and the approach is easy to operate.

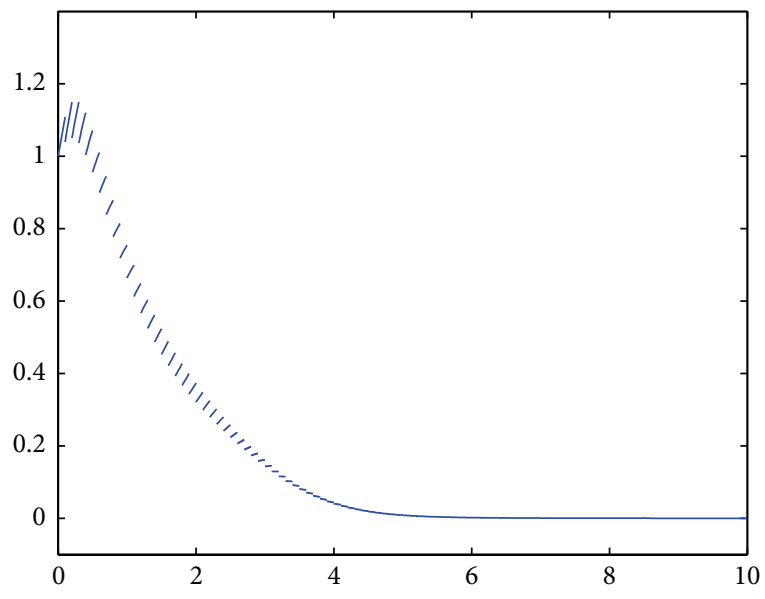

FIGURE 1: Stability of $x(t)$ of system (36).

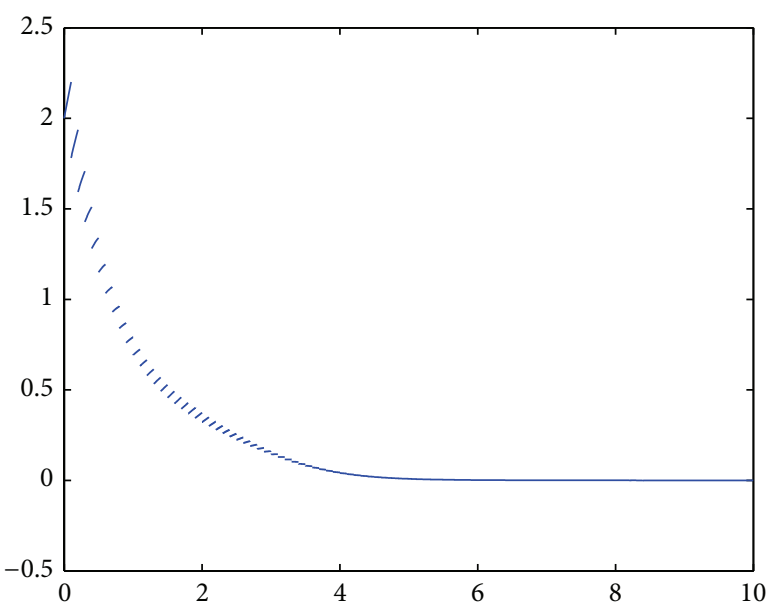

Figure 2: Stability of $y(t)$ of system (36).

Example 3. We consider the system

$$
\begin{aligned}
\frac{d x}{d t}= & \sin (\log (t+1)) y+e^{-t} x \\
& +y(t-\tau) \sin t-\left(x^{3}+x y^{2}\right) \sin ^{2} t, \quad t \neq t_{k}, \\
\frac{d y}{d t}= & \sin (\log (t+1)) x+e^{-t} y+x(t-\tau) \sin t \\
& -\left(x^{2} y+y^{3}\right) \sin ^{2} t, \quad t \neq t_{k}, \quad \\
\Delta x & =a x+b y, \quad \Delta y=b x+a y, \quad t=t_{k},
\end{aligned}
$$

where $2 a=\sqrt{1+c_{1}}+\sqrt{1+c_{2}}-2,2 b=\sqrt{1+c_{1}}-\sqrt{1+c_{2}}$, $-1<c_{1} \leq 0$, and $-1<c_{2} \leq 0$.

Combining Corollary 12 and imitating Example 1, we can obtain the comparison system

$$
\begin{array}{r}
\frac{d u_{1}}{d t}=|\sin (\log (t+1))| u_{2}+e^{-t} u_{1}+|\sin t| u_{2}(t-\tau), \\
t \neq t_{k},
\end{array}
$$




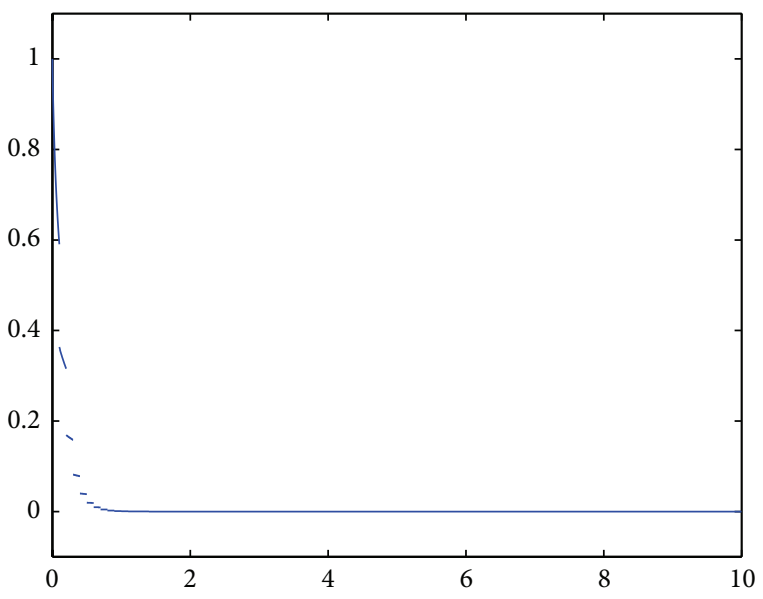

FIGURE 3: Stability of $x(t)$ of system (40).

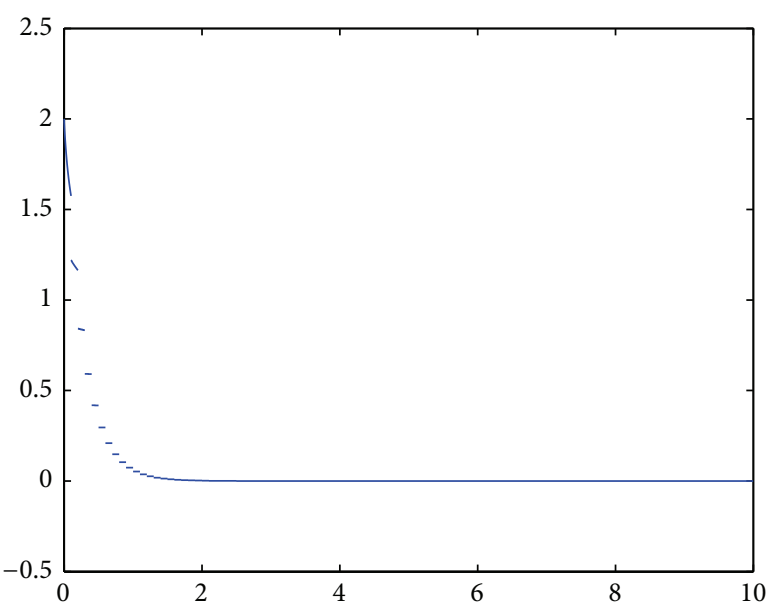

Figure 4: Stability of $y(t)$ of system (40).

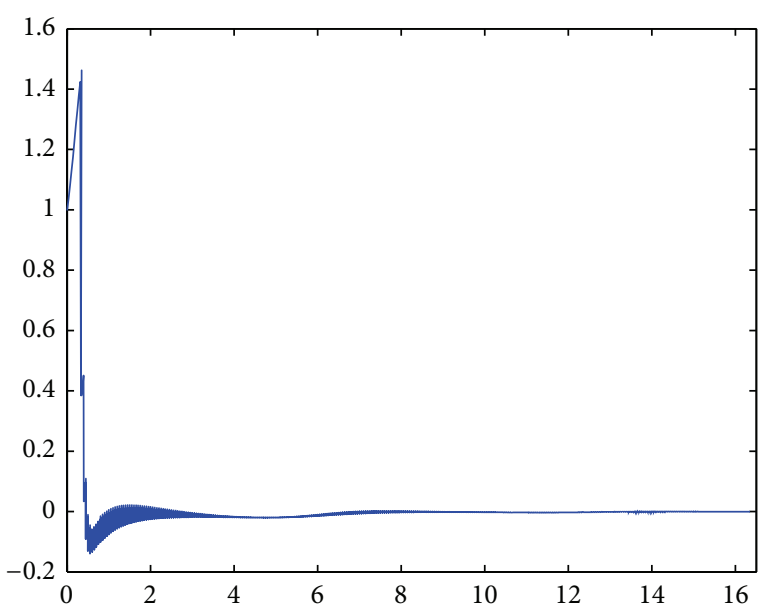

FIgURE 5: Stability of $x(t)$ of system (42).

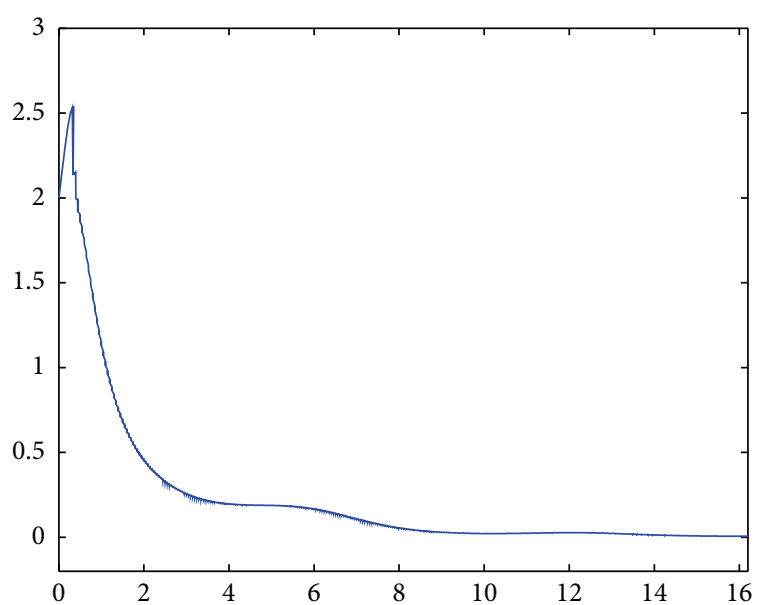

Figure 6: Stability of $y(t)$ of system (42).

$$
\begin{array}{r}
\frac{d u_{2}}{d t}=|\sin (\log (t+1))| u_{1}+e^{-t} u_{2}+|\sin t| u_{1}(t-\tau), \\
t \neq t_{k}, \\
u_{1}\left(t_{k}^{+}\right)=|1+a| u_{1}+|b| u_{2}, \quad u_{2}\left(t_{k}^{+}\right)=|b| u_{1}+|1+a| u_{2}, \\
t=t_{k},
\end{array}
$$

where $u_{1}=|\alpha x+\beta y|, u_{2}=|\alpha y+\beta x|$. When the constants $\alpha, \beta$ satisfy the condition in Theorem 14 , it is true that the zero solution of system (42) is stable. Taking $c_{1}=-0.8, c_{2}=-0.9$, $\tau=1$, and initial conditions $(x, y)^{T}=(1,2)^{T}$, the stability simulations of the states $x(t), y(t)$ of system (42) are as shown in Figures 5 and 6, respectively.

Example 4. We consider the system

$$
\begin{gathered}
\frac{d x}{d t}=-2 x\left(y^{2}-(2+\sin t) z\right)^{2}-\frac{x \cos t}{2+\sin t}, \quad t \neq t_{k}, \\
\frac{d y}{d t}=e^{-t} y+z \sin t-\left(y^{3}+y z^{2}\right) \sin ^{2} t, \quad t \neq t_{k}, \\
\frac{d z}{d t}=y \sin t+e^{-t} z-\left(y^{2} z+z^{3}\right) \sin ^{2} t, \quad t \neq t_{k}, \\
\Delta x=\left(\frac{-1}{2}\right) x, \quad \Delta y=a y+b z, \quad \Delta z=b y+a z, \\
\quad t=t_{k},
\end{gathered}
$$

where $2 a=\sqrt{1+c_{1}}+\sqrt{1+c_{2}}-3,2 b=\sqrt{1+c_{1}}-\sqrt{1+c_{2}}$, $-1<c_{1} \leq 0$, and $-1<c_{2} \leq 0$. 


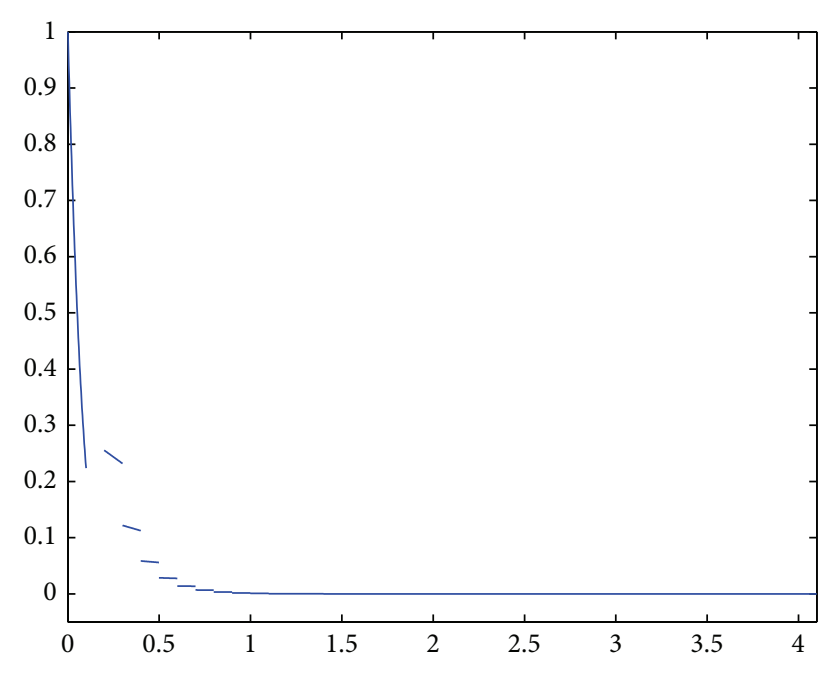

FIGURE 7: Stability of $x(t)$ of system (44).

Combining Corollary 6 or Corollary 13 and imitating Example 1, we can obtain the comparison system

$$
\begin{array}{r}
\frac{d u_{1}}{d t}=\left|\frac{\cos t}{2+\sin t}\right| u_{1}, \quad t \neq t_{k}, \\
\frac{d u_{2}}{d t}=\left|e^{-t}+\sin t\right| u_{2}, \quad t \neq t_{k}, \\
u_{1}\left(t_{k}^{+}\right)=\left(\frac{1}{2}\right)|\alpha| u_{1}, \quad u_{2}\left(t_{k}^{+}\right)=\left\|\left(\begin{array}{cc}
1+a & b \\
b & 1+a
\end{array}\right)\right\| u_{2}, \\
t=t_{k},
\end{array}
$$

where $u_{1}=\left\|\alpha\left(\begin{array}{l}x \\ 0\end{array}\right)\right\|, u_{2}=\left\|\beta\left(\begin{array}{c}y \\ z\end{array}\right)\right\|$, or the comparison system

$$
\begin{gathered}
\frac{d u_{1}}{d t}=\left|\frac{\cos t}{2+\sin t}\right| u_{1}, \quad t \neq t_{k}, \\
\frac{d u_{2}}{d t}=e^{-t} u_{2}+|\sin t| u_{3}, \quad t \neq t_{k}, \\
\frac{d u_{3}}{d t}=|\sin t| u_{2}+e^{-t} u_{3}, \quad t \neq t_{k}, \\
u_{1}\left(t_{k}^{+}\right)=\left(\frac{1}{2}\right) u_{1}, \\
u_{2}\left(t_{k}^{+}\right)=|1+a| u_{2}+|b| u_{3}, \\
u_{3}\left(t_{k}^{+}\right)=|b| u_{2}+|1+a| u_{3}, \\
t=t_{k},
\end{gathered}
$$

where $u_{1}=|\gamma x|, u_{2}=|\alpha y+\beta z|$, and $u_{3}=|\alpha z+\beta y|$. When the constants $\alpha, \beta$, and $\gamma$ satisfy the conditions in Theorem 14 or Remark 15, it is true that the zero solution of system (44) is stable. Taking $c_{1}=-0.2, c_{2}=-0.5$, and initial conditions $(x, y, z)^{T}=(1,2,0.8)^{T}$, the stability simulations of the states $x(t), y(t)$, and $z(t)$ of system (44) are as shown in Figures 7, 8 , and 9 , respectively.
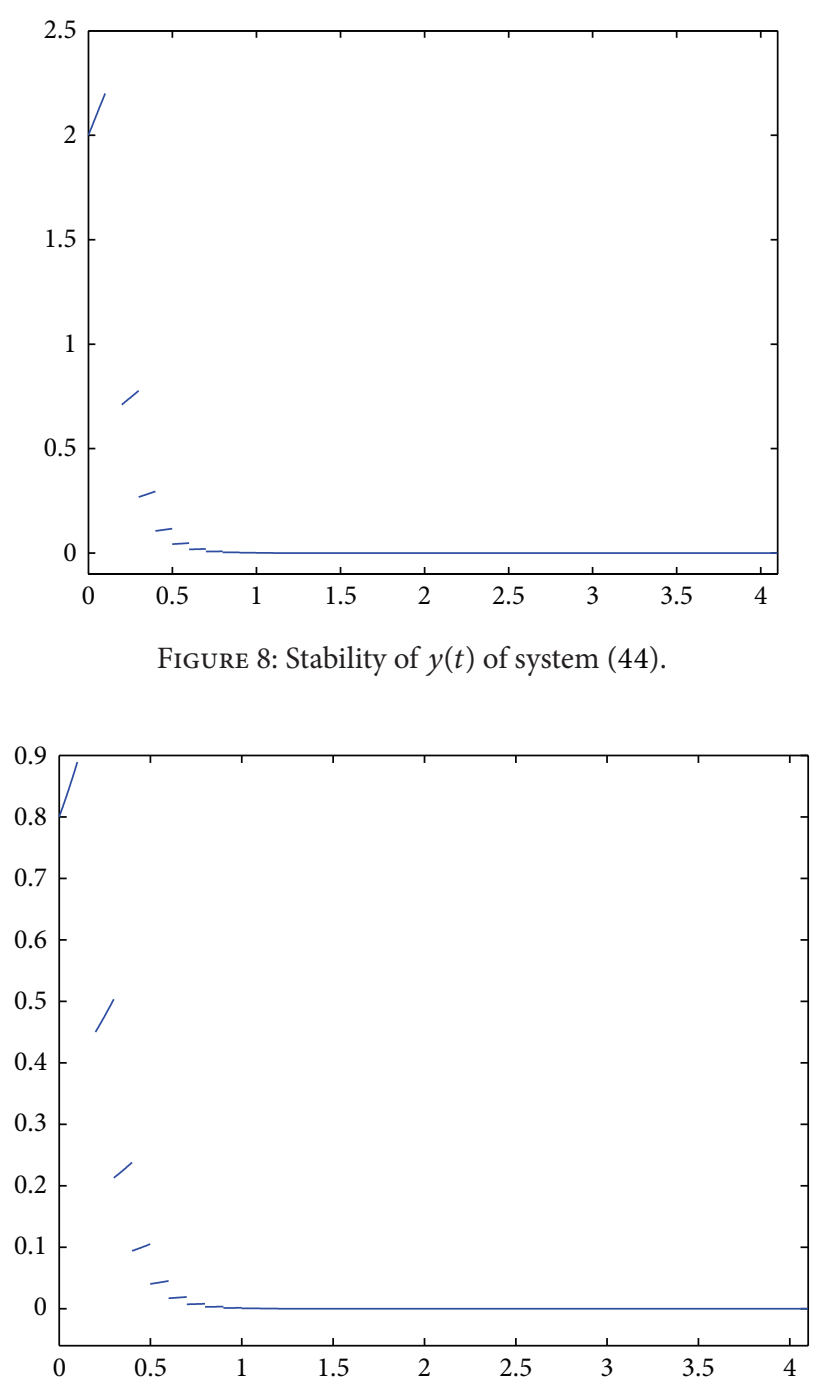

FIGURE 9: Stability of $z(t)$ of system (44).

\section{Conclusion}

This letter, which uses a class of vector Lyapunov functions, has studied the issue on impulsive stability of nonlinear systems and improves the existing results. Numerical simulations have verified the effectiveness of the method.

\section{Conflict of Interests}

The author declare that there is no conflict of interests regarding the publication of this paper.

\section{Acknowledgments}

The author was supported financially by the National Natural Science Foundation of China (11071141), the Natural Science Foundation of Shandong Province of China (ZR2011AL018, ZR2011AQ008), the Project of Shandong Province Higher Educational Science and Technology Program (J11LA06, J13LI02), and the Research Fund Project of Heze University (XY10KZ01). 


\section{References}

[1] D. D. Bainov and P. S. Simeonov, "Stability with respect to part of the variables in systems with impulse effect," Journal of Mathematical Analysis and Applications, vol. 124, no. 2, pp. 547560, 1987.

[2] A. M. Samoilenko and N. A. Perestyuk, Differential Equations with Impulsive Effect, Visca Skola, Kiev, Ukraine, 1987, (Russian).

[3] G. K. Kulev and D. D. Bainov, "Second method of Lyapunov and comparison principle for systems with impulse effect," Journal of Computational and Applied Mathematics, vol. 23, no. 3, pp. 305-321, 1988.

[4] D. D. Bainov and P. S. Simeonov, Systems with Impulsive Effect: Stability Theory and Applications, Ellis Horwood, Chichester, UK, 1989.

[5] D. D. Bainov and P. S. Simeonov, Theory of Impulsive Differential Equations: Periodic Solutions and Applications, Longman, Harlow, UK, 1993.

[6] L. O. Chua, M. Itoh, L. Kocare, and K. Eckert, "Chaos synchronization in Chua's circuit," Journal of Circuits, Systems and Computers, vol. 3, no. 1, pp. 93-108, 1993.

[7] E. R. Hunt and G. Johnson, "Keeping chaos at bay," IEEE Spectrum, vol. 30, no. 11, pp. 32-36, 1993.

[8] M. Itoh, T. Yang, and L. O. Chua, "Experimental study of impulsive synchronization of chaotic and hyperchaotic circuits," International Journal of Bifurcation and Chaos in Applied Sciences and Engineering, vol. 9, no. 7, pp. 1393-1424, 1999.

[9] H. Wei and Z. Hua-Guang, "Control of pulse synchronization for a class of chaotic systems," Journal of Northeastern University: Natural Science, vol. 25, no. 11, pp. 1027-1029, 2004.

[10] S. Mei and T. Li-Xin, "Adaptive synchronization of non-linear chaotic finance system," Joural of Jiangsu University: Natural Science Edition, vol. 26, pp. 488-491, 2005.

[11] I. M. Stamova, "Vector Lyapunov functions for practical stability of nonlinear impulsive functional differential equations," Journal of Mathematical Analysis and Applications, vol. 325, no. 1, pp. 612-623, 2007.

[12] Z. Qunli, "Synchronization of multi-chaotic systems via ring impulsive control," Control Theory and Applications, vol. 27, no. 2, pp. 226-232, 2010.

[13] S. Xinyu, G. Hongjian, and S. Xiangyun, Theory and Application of Impulsive Differential Equations, Science Press, Beijing, China, 2011.

[14] Z. Qunli, “The generalized dahlquist constant with applications in synchronization analysis of typical neural networks via general intermittent control," Advances in Artificial Neural Systems, vol. 2011, Article ID 249136, 7 pages, 2011.

[15] Z. Qunli, "Generalized Dahlquist constant with applications in synchronization analysis excited by parameter white-noise of typical neural networks," Annals of Differential Equations, vol. 28, no. 14, pp. 480-487, 2012.

[16] M. Han, Y. Liu, and J. Lu, "Impulsive control for the synchronization of chaotic systems with time delay," Abstract and Applied Analysis, vol. 2012, Article ID 647561, 13 pages, 2012.

[17] K. Zhang and X. Liu, "Stability in terms of two measures for nonlinear impulsive systems on time scales," Journal of Applied Mathematics, vol. 2013, Article ID 313029, 12 pages, 2013.

[18] Q. Song and J. Cao, "Stability analysis of Cohen-Grossberg neural network with both time-varying and continuously distributed delays," Journal of Computational and Applied Mathematics, vol. 197, no. 1, pp. 188-203, 2006.
[19] X. Liao, Theory and Application of Stability for Dynamical Systems, National Defence Industry Press, Beijing, China, 2000.

[20] P. S. Simeonov and D. D. Bainov, "Stability with respect to part of the variables in systems with impulse effect," Journal of Mathematical Analysis and Applications, vol. 117, no. 1, pp. 247263, 1986.

[21] K.-M. Wang, X.-L. Hu, and S.-Y. Jia, "Nonlinear measure about $1^{2}$-norm," Basic Sciences Journal of Texttile Universitties, vol. 17, no. 3, pp. 198-200, 2004.

[22] H. Lu, Z. Zhang, and L. He, "Convergence analysis of genetic regulatory networks based on nonlinear measures," in Proceedings of the 2nd International Symposium on Neural Networks: Advances in Neural Networks (ISNN '05), pp. 247-252, June 2005.

[23] P. Li and J. Cao, "Stability in static delayed neural networks: a nonlinear measure approach," Neurocomputing, vol. 69, no. 1315, pp. 1776-1781, 2006.

[24] A. Wan, M. Wang, and J. Peng, "Exponential stability of CohenGrossberg neural networks," Journal of Xi'an Jiaotong University, vol. 40, no. 2, pp. 215-218, 2006.

[25] P. Li and J. Cao, "Global stability in switched recurrent neural networks with time-varying delay via nonlinear measure," Nonlinear Dynamics, vol. 49, no. 1-2, pp. 295-305, 2007.

[26] Y. Li and H. Jiang, "Exponential stability of high-order CohenGrossberg neural networks with time delay," Journal of Xinjiang University: Natural Science Edition, vol. 25, no. 1, pp. 28-35, 2008. 


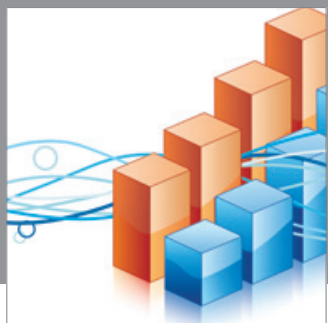

Advances in

Operations Research

mansans

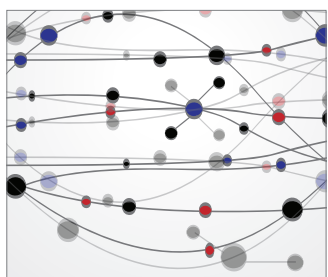

The Scientific World Journal
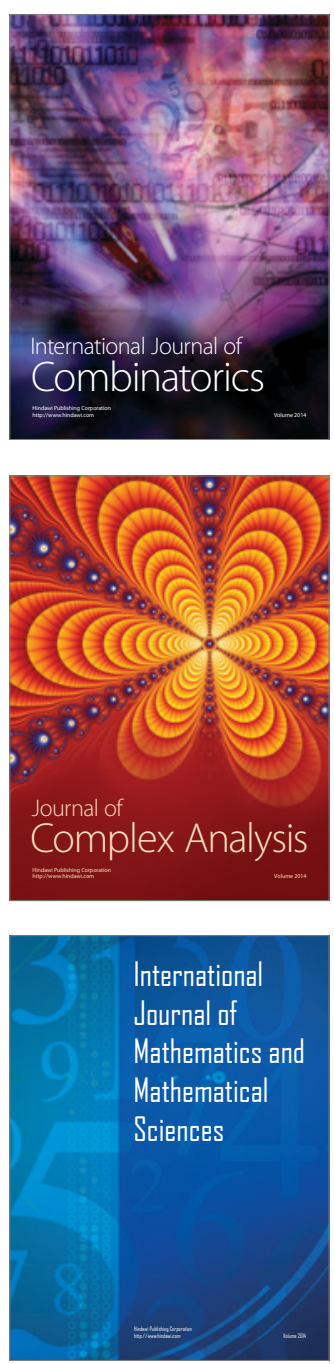
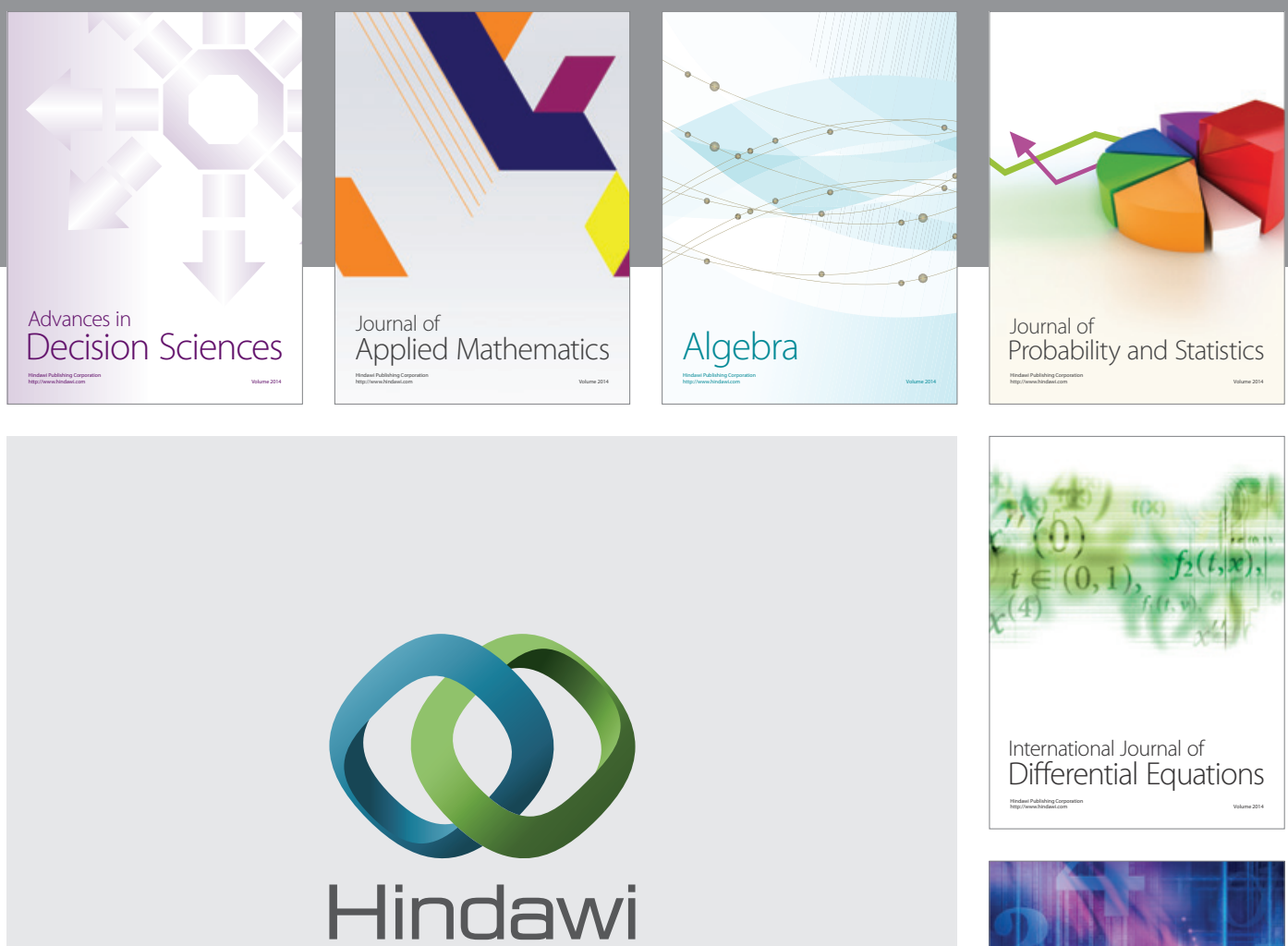

Submit your manuscripts at http://www.hindawi.com
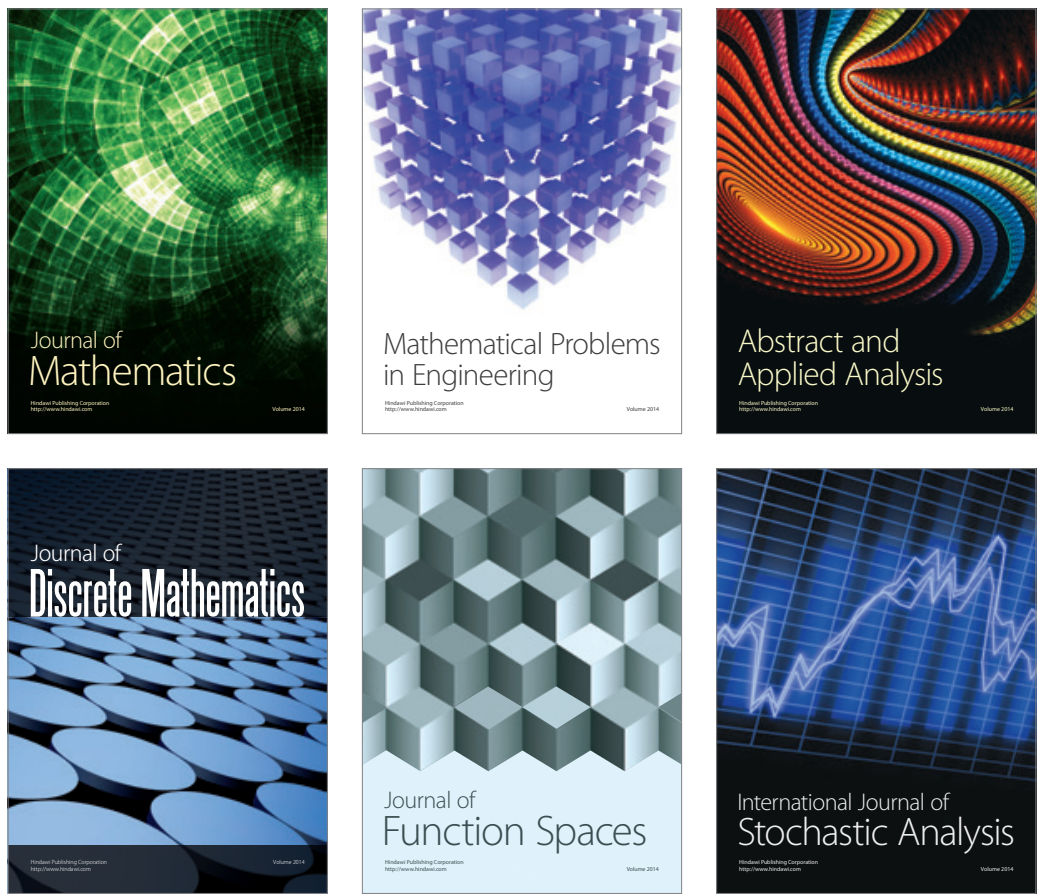

Journal of

Function Spaces

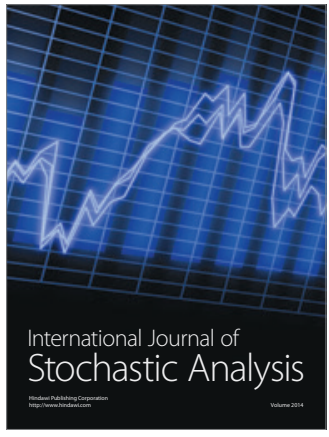

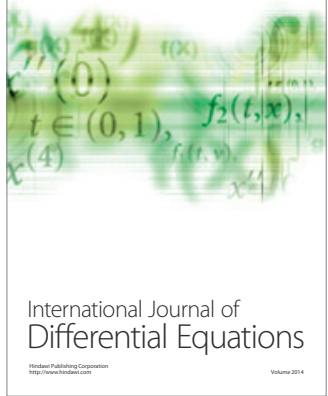
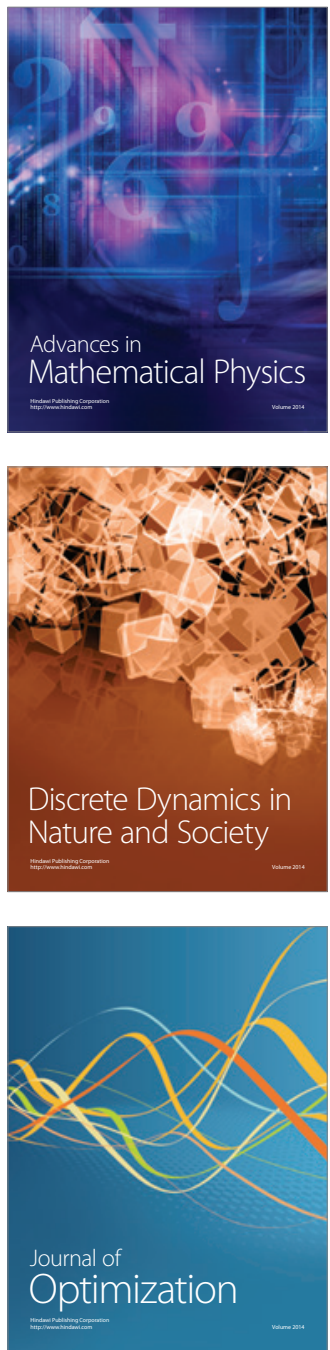